\title{
Vegan men have less stiff arteries despite the lack of dietary docosahexaenoic acid and eicosapentaenoic acid
}

\author{
J. Taylor and T. A. B. Sanders \\ Diabetes and Nutritional Sciences Division, King's College London, 150 Stamford Street, London SE1 9NH, UK
}

\begin{abstract}
Arterial stiffness is an index of the aging of large conduit arteries. Fish consumption, which is associated with higher dietary intakes of docosahexaenoic (22:6n-3; DHA) and eicosapentaenoic (20:5n-3EPA) acids, has been linked to reduced arterial stiffness ${ }^{(1)}$. We tested the hypothesis that vegans, who eat no fish or other sources of EPA and DHA, would have stiffer arteries in comparison to omnivores. We extracted data previously collected in our laboratory from two studies ${ }^{(2,3)}$ on 171 vegan and 165 omnivore healthy men ranging in age from

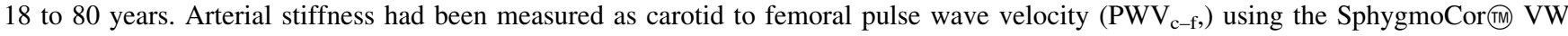
and software (SphygmoCor $\mathrm{IM}$ version 7.01 AtCor Medical Pty, Australia) employing an identical protocol conducted by experienced staff over the same time period (2000-2003). Data were available for supine systolic and diastolic blood pressure prior to measurement of $\mathrm{PWV}_{\mathrm{c}-\mathrm{f}}$, and plasma total fatty acid composition and the serum lipid profile had been measured using identical methods in the same laboratories on fasting samples collected at the same time as the $\mathrm{PWV}_{\mathrm{c}-\mathrm{f}}$ measurement. As the vegan subjects were younger and had a lower body mass index (BMI) than the omnivores, we compared vegan men matched by age and BMI with omnivore controls. We matched 64 pairs aged $50 \pm 11 \mathrm{y}$ (mean \pm SD) and BMI $24.4 \pm 2.9 \mathrm{~kg} / \mathrm{m}^{2}$. Data were analysed using the SPSS version 20 package (SPSS Inc Chicago). Results are shown in the Table.
\end{abstract}

\begin{tabular}{|c|c|c|c|c|}
\hline & \multicolumn{2}{|c|}{ Vegans $n 64$} & \multicolumn{2}{|c|}{ Omnivores $n 64$} \\
\hline & Mean & SD & Mean & SD \\
\hline Plasma EPA, wt $\%^{\dagger}$ & $0.42 * *$ & 0.37 & 1.40 & 0.86 \\
\hline Plasma DHA, wt $\%^{\dagger}$ & $0.85^{* *}$ & 0.31 & 2.77 & 1.01 \\
\hline LDL-C, $\mathrm{mmol} / \mathrm{L}$ & $2.57 * *$ & 0.66 & 3.09 & 0.74 \\
\hline $\mathrm{HDL}-\mathrm{C}, \mathrm{mmol} / \mathrm{L}$ & 1.27 & 0.32 & 1.38 & 0.36 \\
\hline Supine systolic BP, mmHg & 121 & 15 & 124 & 18 \\
\hline Supine diastolicBP, $\mathrm{mmHg}$ & $72 *$ & 9 & 75 & 9 \\
\hline $\mathrm{PWV}_{\mathrm{c}-\mathrm{f}}, \mathrm{m} / \mathrm{sec}$ & $8.25 *$ & 1.59 & 8.88 & 2.24 \\
\hline
\end{tabular}

The proportions of EPA and DHA in the plasma of vegans were only a third of those in omnivores, and LDL-C and diastolic BP were $18 \%$ and $3 \mathrm{~mm} \mathrm{Hg}$ lower respectively. Arterial stiffness measured as $\mathrm{PWV}_{\mathrm{c}-\mathrm{f}}$ was $0.63(95 \% \mathrm{CI} 0.09,1.17 ; P=0.023) \mathrm{m} / \mathrm{sec} l \mathrm{lower}$ in the vegans compared to the omnivores. In conclusion, these results refute the hypothesis that arterial stiffness is increased by a lack of fish and other sources of EPA and DHA in the vegan diet. It may well be that the consequences of lack of EPA and DHA are mitigated by other aspects of the vegan diet i.e. a more favourable lipid profile, lower BMI and blood pressure. Indeed, there was tentative evidence to suggest that arterial ageing was slower in the vegans.

1. Mozaffarian D, Wu JH (2012) J Nutr 142, 614S-625S.

2. Lloyd-Wright Z, Hvas AM, Moller J, Sanders TA, Nexo E (2003) Clin Chem 49, 2076-2078

3. Sanders TA, Lewis F, Slaughter S, Griffin BA, Griffin M, Davies I, Millward DJ, Cooper JA, Miller GJ. (2006) Am J Clin Nutr 84, 513-22. 